Alessandra Gutierrez de Oliveira 1 José Dilermando Andrade Filho 2 Alda Lima Falcão 2 Reginaldo Peçanha Brazil 3

\section{Estudo de flebotomíneos (Diptera, Psychodidae, Phlebotominae) na zona urbana da Cidade de Campo Grande, Mato Grosso do Sul, Brasil, 1999-2000}

\author{
Study of sand flies (Diptera, Psychodidae, \\ Phlebotominae) in the urban area \\ of Campo Grande, Mato Grosso do Sul State, \\ Brazil, from 1999 to 2000
}

1 Centro de Controle de Zoonoses, Secretaria Municipal de Saúde de Campo Grande. Av. Filinto Muller 1601 Campo Grande, MS 79074-460, Brasil. rlemos@nin.ufms.br 2 Centro de Pesquisas René Rachou, Fundação Oswaldo Cruz. Av. Augusto de Lima 1715, Belo Horizonte, $M G$ 30190-002, Brasil. 3 Departamento de Bioquímica e Biologia Molecular, Instituto Oswaldo Cruz, Fundação Oswaldo Cruz. Av. Brasil 4365, Rio de Janeiro, $R J$ 21045-900, Brasil. rpbrazil@ioc.fiocruz.br

\begin{abstract}
From February 1999 to February 2000, sand flies were captured weekly with CDC light traps at five sites in the urban area of Campo Grande, Mato Grosso do Sul State, Brazil. Traps were placed in 11 different ecotopes in the environment (ground level, tree canopies, and forest edge) and the peridomicile (chicken coops and banana trees). A total of 1,245 sand flies were captured, belonging to 28 species: 4 species from genus Brumptomyia França \& Parrot, 1921 and 24 from genus Lutzomyia França, 1924. The species were: B. avellari, B. brumpti, B. galindoi, B. pintoi, L. aragaoi, L. bourrouli, L. campograndensis, L. cerradincola, L. christenseni, L. claustrei, L. cortelezzii, L. corumbaensis, L. cruzi, L. damascenoi, L. flaviscutellata, L. hermanlenti, L. lenti, L. longipalpis, L. longipennis, L. migonei, L. punctigeniculata, L. quinquefer, L. renei, L. shannoni, L. sordellii, L. teratodes, L. termitophila, and L. whitmani. L. longipalpis and L. cruzi, vectors of visceral leishmaniasis, and L. whitmani, L. flaviscutellata and L. migonei, vectors of cutaneous leishmaniasis, were captured in the urban area. The most frequent species were L. termitophila, L. aragaoi, L. lenti, L. longipennis, and L. longipalpis.

Key words Leishmaniasis; Sandflies; Vector Control
\end{abstract}

Resumo De fevereiro de 1999 a fevereiro de 2000, realizaram-se capturas semanais com armadilhas luminosas do tipo CDC em cinco regiões da zona urbana da Cidade de Campo Grande, Estado de Mato Grosso do Sul, Brasil. As armadilhas foram colocadas em 11 ecótopos (copa, solo e margem nas matas) e nos peridomicílios (galinheiro e bananeiras). Foram capturados 1.245 flebotomíneos de 28 espécies, sendo 4 do gênero Brumptomyia França \& Parrot, 1921 e 24 do gênero Lutzomyia França, 1924. São elas: B. avellari, B. brumpti, B. galindoi, B. pintoi, L. aragaoi, L. bourrouli, L. campograndensis, L. cerradincola, L. christenseni, L. claustrei, L. cortelezzii, L. corumbaensis, L. cruzi, L. damascenoi, L. flaviscutellata, L. hermanlenti, L. lenti, L. longipalpis, L. longipennis, L. migonei, L. punctigeniculata, L. quinquefer, L. renei, L. shannoni, L. sordellii, L. teratodes, L. termitophila $e \mathrm{~L}$. whitmani. Destaca-se a presença em área urbana de vetores da leishmaniose visceral (L. longipalpis $e$ L. cruzi) e da leishmaniose tegumentar (L. whitmani, L. flaviscutellata $e$ L. migonei). As cinco espécies mais freqüentes foram: L. termitophila, L. aragaoi, L. lenti, L. longipennis $e$ L. longipalpis.

Palavras-chave Leishmaniose; Flebotomíneos; Controle de Vetores 


\section{Introdução}

Segundo relatórios fornecidos pelo SINAN (Sistema de Informações de Agravos de Notificação), Serviço de Vigilância Epidemiológica, Secretaria Estadual de Saúde de Mato Grosso do Sul, no período compreendido entre 1995 e 2000, foram notificados 2.330 casos de Leishmaniose Tegumentar Americana (LTA), abrangendo 74 municípios do Estado. Em 2002 foram notificados 106 casos de Leishmaniose Visceral Americana (LVA), com sete óbitos, abrangendo 13 municípios, entre estes, os municípios de Três Lagoas com 55 casos confirmados e três óbitos e Campo Grande, capital do Estado, que até então só tem apresentado casos importados de outros municípios. Atualmente, a situação é preocupante, pois já foram notificados, até o mês de maio de 2002, mais casos em Três Lagoas do que nas áreas do Estado reconhecidamente endêmicas para LVA.

Ressalta-se que, sendo a economia de Mato Grosso do Sul baseada na agropecuária, pode-se correlacionar a ocorrência de casos de leishmanioses com a implantação de projetos agropecuários, abertura de estradas e o assentamento de trabalhadores rurais sem-terra, sem preocupação com infra-estrutura sanitária, como observado em outras regiões do Brasil (Azevedo et al., 1996; Mayrink et al., 1979).

$\mathrm{O}$ crescimento da cidade, devido à situação geográfica de Campo Grande, que se posiciona como entrada obrigatória de migrantes para o norte do País e a atração exercida pela capital que se encontra em expansão administrativa e econômica, faz com que seja preocupante a introdução da LVA na cidade, pois não há controle da circulação de cães possivelmente portadores da doença.

Este trabalho baseia-se em um levantamento da fauna flebotomínea da zona urbana da cidade de Campo Grande, na tentativa de identificar, determinar a sua ocorrência, distribuição e densidade, com a finalidade de fornecer subsídios a programas futuros de controle das leishmanioses no Estado.

\section{Material e métodos}

\section{Área de estudo}

\section{- Características gerais}

O Município de Campo Grande, Mato Grosso do Sul, Brasil, com $8.118,4 \mathrm{~km}^{2}$, está localizado geograficamente na porção central do Estado, ocupando $2,27 \%$ de sua área total e, segundo a
Fundação Instituto Brasileiro de Geografia e Estatística (IBGE, 1991), com uma população urbana de 518.687 habitantes.

A sede do município localiza-se nas imediações do divisor de águas das bacias do Paraná e Paraguai, definida pelas coordenadas geográficas $20^{\circ} 26^{\prime} 34^{\prime \prime}$ latitude Sul e 54³8'47" longitude Oeste.

O município está na unidade litológica da Bacia Sedimentar do Paraná no compartimento denominado Planalto de Maracajú-Campo Grande, assentado nas formações Caiuá do Grupo Bauru e Serra Geral e Botucatu do Grupo São Bento. Localiza-se na zona neotropical, pertencente aos domínios da região fitogeográfica de Savana. Sua cobertura vegetal autóctone apresenta-se com as fisionomias de Savana Arbórea Densa, Savana Arbórea Aberta, Savana Parque e Savana Gramíneo Lenhosa (Campo limpo), denominado de Campo de Vacaria, que é formado predominantemente pelo estrato herbáceo e por algumas espécies subarbustivas (Prefeitura Municipal de Campo Grande, 1995).

No perímetro urbano durante o período de ocupação, houve a retirada da cobertura vegetal autóctone, sendo substituída posteriormente pela arborização por espécies que nada retratam a realidade da flora regional.

Nos corpos d'água localizados na área urbana, há a presença de extensas áreas ocupadas por veredas e de alguns remanescentes florestais nas nascentes, dentre estes, a Reserva Ecológica do Parque dos Poderes e o Jardim Botânico.

O clima predominante de Campo Grande, conforme classificação de Köppen, é do tipo tropical chuvoso de Savana, subtipo AW, caracterizado por má distribuição anual das chuvas, com ocorrência bem definida de um período seco durante os meses mais frios do ano e um período chuvoso durante os meses de verão. A temperatura média anual é em torno de $23^{\circ} \mathrm{C}$; dezembro é o mês mais quente, aproximadamente $25^{\circ} \mathrm{C}$, e junho o mais frio, com $18^{\circ} \mathrm{C}$ em média.

\section{- Características locais}

Foram escolhidas cinco regiões na área urbana de acordo com a divisão setorial estipulada pela prefeitura. São elas: Norte, Sul, Oeste, Leste e Central. Respectivamente: Jardim Botânico, Chácara das Palmeiras, Mata do Zé Pereira, Reserva Ecológica do Parque dos Poderes (CRAS Centro de Reabilitação de Animais Silvestres) e uma residência (Figura 1).

No Jardim Botânico, existe uma reserva ecológica com 170ha, onde se encontra a nas- 
Área urbana do Município de Campo Grande, Mato Grosso do Sul, Brasil e os respectivos pontos de coletas: 1. Mata do Zé Pereira; 2. Jardim Botânico; 3. Centro de Reabilitação de Animais Silvestres; 4. Centro; 5. Chácara das Palmeiras.

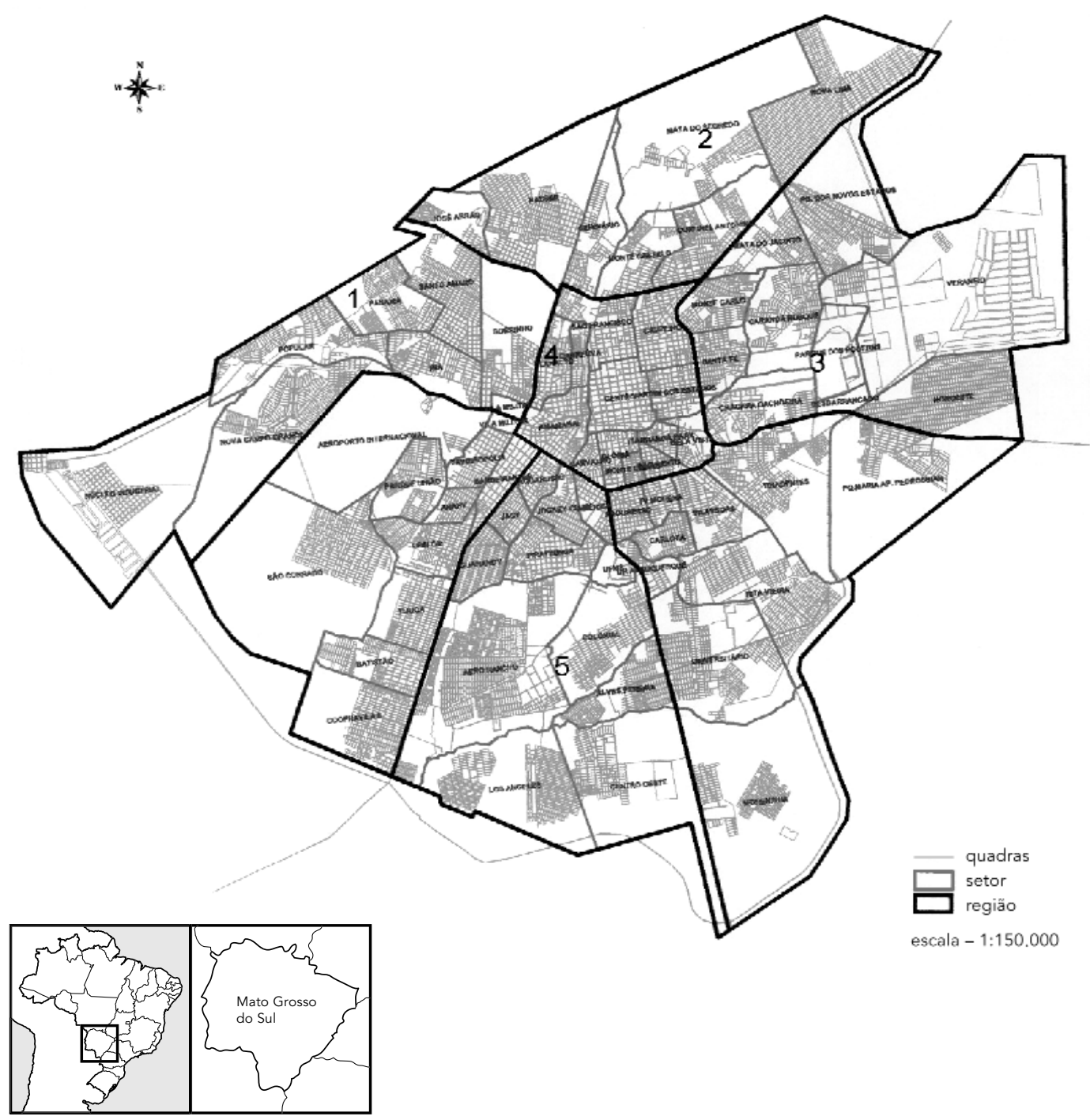

cente do Córrego Segredo, composta por vegetação nativa apresentando árvores com mais de $5 \mathrm{~m}$ de altura; possui um posto avançado da polícia florestal e atualmente sedia um grupamento infantil que faz treinamentos inclusive com pernoite na mata.

A Chácara das Palmeiras pertence a uma veterinária do Centro de Controle de Zoonoses (CCZ) e compreende um terreno de um hectare com duas residências de alvenaria, pomar com grande diversidade de espécies, canil, criação de caprinos e um grande galinheiro que faz divisa com uma faixa de mata residual no terreno vizinho.

A Mata do Zé Pereira está localizada em um bairro da periferia que foi criado por um programa de desfavelamento, compreendendo uma faixa de mata remanescente em que se observam fauna e flora ricas; entretanto, os moradores do local jogam lixo e animais mortos, servindo, ainda, de abrigo para desocupados.

O CRAS faz parte da Reserva Ecológica do Parque dos Poderes, com aproximadamente 120ha, é composta por mata nativa e fechada, 
com grande diversidade de fauna e flora. A área é considerada um dos pontos turísticos da cidade com grande visitação turística.

A residência na região Central é construída em madeira e apresenta uma área com cerca de $2.500 \mathrm{~m}^{2}$, com cultura diversificada de frutas, destacando-se bananeiras.

\section{Capturas}

As coletas foram realizadas com armadilhas automáticas luminosas do tipo CDC, instaladas semanalmente, das $18 \mathrm{~h}$ às $6 \mathrm{~h}$, no período compreendido entre fevereiro de 1999 e fevereiro de 2000, perfazendo um total de 53 semanas. Foram 11 os ecótopos selecionados: Jardim Botânico (solo, copa e margem da mata), Mata do Zé Pereira (solo, copa e margem), CRAS (solo, copa e margem), galinheiro situado no peridomicílio de casa na Chácara das Palmeiras, plantações de bananas localizadas no peridomicílio de casa na Região Central. As armadilhas das copas foram instaladas aproximadamente a $5 \mathrm{~m}$ de altura e as armadilhas do solo e margem a $1 \mathrm{~m}$. Deve ser ressaltado que, para padronizar o número de armadilhas em cada região, optou-se por colocar três armadilhas no galinheiro e no peridomicílio.

Os cálculos do índice de diversidade foram feitos segundo o método de Margalef (Margalef, 1974; Service, 1993).

Para a avaliação da atividade das espécies mais abundantes dos flebotomíneos, utilizouse a média de Williams (Haddow, 1954, 1960) por coleta semanal, tendo em vista que essa medida de tendência central reflete não somente a freqüência das espécies nas coletas, mas também a regularidade nas mesmas. Também foi utilizada a média aritmética para todas as espécies, em todos os ecótopos.

Foram utilizados os dados meteorológicos da estação da Empresa Brasileira de Pesquisa Agropecuária - EMBRAPA (CNPGC - Centro Nacional de Pesquisa de Gado de Corte), em Campo Grande, Mato Grosso do Sul, Brasil.

Os flebotomíneos capturados foram fixados em álcool 70\% e levados ao Laboratório de Entomologia do CCZ onde foram preparados e montados entre lâmina e lamínula em líquido de Berlese. A identificação foi feita de acordo com Young \& Duncan (1994).

\section{Resultados}

Nas 265 capturas realizadas ao longo de um ano, foi coletado um total de 1.245 flebotomíneos pertencentes a 28 espécies; destas, quatro estão inseridas no gênero Brumptomyia França \& Parrot e 24 no gênero Lutzomyia França. São elas: B. avellari (Costa Lima, 1932), B. brumpti (Larrousse, 1920), B. galindoi (Fairchild \& Hertig, 1947), B. pintoi (Costa Lima, 1932), L. aragaoi (Costa Lima, 1932), L. bourrouli (Barretto \& Coutinho, 1941), L. campograndensis Oliveira, Andrade Filho, Falcão \& Brazil, 2001, L. cerradincola Galati, Nunes, Oshiro \& Dorval, 1995, L. christenseni Young \& Duncan, 1994, L. claustrei Abonnenc Léger \& Fauran, 1979, L. cortelezzii (Brèthes, 1923), L. corumbaensis Galati, Nunes, Oshiro \& Rego, 1989, L. cruzi (Mangabeira, 1938), L. damascenoi (Mangabeira, 1941), L. flaviscutellata (Mangabeira, 1942), L. hermanlenti Martins, Silva \& Falcão, 1970, L. lenti (Mangabeira, 1938), L. longipalpis (Lutz \& Neiva, 1912), L. longipennis (Barretto, 1946), L. migonei (França, 1920), L. punctigeniculata (Floch \& Abonnenc, 1944), L. quinquefer (Dyar, 1929), L. renei (Martins, Falcão \& Silva, 1957), L. shannoni (Dyar, 1929), L. sordellii (Shannon \& Del Ponte, 1927), L. teratodes (Martins, Falcão \& Silva, 1964), L. termitophila Martins, Falcão \& Silva, 1964, L. whitmani (Antunes \& Coutinho, 1939) (Tabela 1).

A maior diversidade foi obtida na Chácara das Palmeiras com 22 espécies (Índice de diversidade $=3,51$ ) seguida pela Mata do Zé Pereira com vinte espécies ( $D=3,33$ ), CRAS com 16 espécies $(\mathrm{D}=2,95)$, Jardim Botânico com 14 espécies $(\mathrm{D}=2,60)$ e a menor, no Centro, com apenas quatro espécies $(D=0,88)$. A espécie com maior densidade foi $L$. termitophila com predominância em galinheiro. Apenas L. lenti foi a espécie que esteve representada em todas as áreas trabalhadas, demostrando que está distribuída em toda a cidade. L. aragaoi, a segunda mais freqüente, apareceu em quatro regiões (Mata do Zé Pereira, CRAS, Jardim Botânico e Chácara das Palmeiras). L. shannoni, B. avellari, L. damascenoi, L. christenseni e L. whitmani também foram encontradas nestas quatro regiões. L. sordellii foi assinalada em quatro regiões e diferiu das anteriores por estar presente no Centro e não no CRAS. L. cortelezzii, também representada em quatro regiões (Mata do Zé Pereira, CRAS, Chácara das Palmeiras e Centro), esteve ausente no Jardim Botânico.

A razão de sexo machos/fêmeas para as espécies mais numerosas foi de 0,78 para $L$. termitophila, 1,12 para L. aragaoi, 1,02 para L. len$t i, 0,87$ para L. longipennis e 3,06 para L. shannoni (Tabela 2).

Na Tabela 3 estão representadas as espécies coletadas e os respectivos meses em que estas foram assinaladas, evidenciando que janeiro de 2000 apresentou o maior rendimento, uma 
Freqüência das espécies de flebotomíneos coletados com CDC, no Jardim Botânico, Centro de Reabilitação de Animais Silvestres, Mata do Zé Pereira, Chácara das Palmeiras e Região Central, Campo Grande, Mato Grosso do Sul, Brasil, fevereiro de 1999 a fevereiro de 2000.

\begin{tabular}{|c|c|c|c|}
\hline Espécies & $\mathbf{n}$ & $\%$ & Acumulado (\%) \\
\hline B. avellari (Costa Lima, 1932) & 56 & 4,5 & 4,5 \\
\hline B. brumpti (Larrousse, 1920) & 44 & 3,5 & 8,0 \\
\hline B. galindoi (Fairchild \& Hertig, 1947) & 54 & 4,3 & 12,4 \\
\hline B. pintoi (Costa Lima, 1932) & 8 & 0,6 & 13,0 \\
\hline Brumptomyia sp. (França \& Parrot, 1921) & 150 & 12,0 & 25,1 \\
\hline L. aragaoi (Costa Lima, 1932) & 125 & 10,0 & 35,1 \\
\hline L. bourrouli (Barreto \& Coutinho, 1941) & 5 & 0,4 & 35,5 \\
\hline $\begin{array}{l}\text { L. campograndensis Oliveira, Andrade Filho, } \\
\text { Falcão \& Brazil, } 2001\end{array}$ & 29 & 2,3 & 37,8 \\
\hline L. cerradincola Galati, Nunes, Oshiro \& Dorval, 1995 & 1 & 0,1 & 37,9 \\
\hline L. christenseni Young \& Duncan, 1994 & 16 & 1,3 & 39,2 \\
\hline L. claustrei Abonnenc, Léger \& Fauran, 1979 & 17 & 1,4 & 40,6 \\
\hline L. cortelezzii (Brèthes, 1923) & 42 & 3,4 & 43,9 \\
\hline L. corumbaensis Galati, Nunes, Oshiro \& Rego, 1989 & 4 & 0,3 & 44,3 \\
\hline L. cruzi (Mangabeira, 1938) & 1 & 0,1 & 44,3 \\
\hline L. damascenoi? (Mangabeira, 1941) & 30 & 2,4 & 46,7 \\
\hline L. flaviscutellata (Mangabeira, 1942) & 6 & 0,5 & 47,2 \\
\hline L. hermanlenti Martins, Silva \&Falcão, 1970 & 45 & 3,6 & 50,8 \\
\hline L. lenti (Mangabeira, 1938) & 117 & 9,4 & 60,2 \\
\hline L. longipalpis (Lutz \& Neiva, 1912) & 71 & 5,7 & 65,9 \\
\hline L. longipennis (Barreto, 1946) & 105 & 8,4 & 74,4 \\
\hline L. migonei (França, 1920) & 1 & 0,1 & 74,5 \\
\hline L. punctigeniculata (Floch \& Abonnenc, 1944) & 4 & 0,3 & 74,8 \\
\hline L. quinquefer (Dyar, 1929) & 10 & 0,8 & 75,6 \\
\hline L. renei (Martins, Falcão \& Silva, 1957) & 1 & 0,1 & 75,7 \\
\hline L. shannoni (Dyar, 1929) & 65 & 5,2 & 80,9 \\
\hline L. sordellii (Shannon \& Del Ponte, 1927) & 44 & 3,5 & 84,4 \\
\hline L. teratodes Martins, Falcão \& Silva, 1964 & 12 & 1,0 & 85,4 \\
\hline L. termitophila Martins, Falcão \& Silva, 1964 & 175 & 14,1 & 99,4 \\
\hline L. whitmani (Antunes \& Coutinho, 1939) & 7 & 0,6 & 100,0 \\
\hline Total & 1.245 & 100,0 & \\
\hline
\end{tabular}

vez que, das 28 espécies encontradas, 21 apareceram neste mês, totalizando 326 flebotomíneos e uma média semanal de 81,50. O mês de dezembro de1999 também foi de bom rendimento, dado que foram coletados 209 exemplares, distribuídos em 18 espécies, com média semanal de 52,25.

Estão plotados na Figura 2 a média de Williams mensal de espécimens de flebotomíneos, de fevereiro de 1999 a fevereiro de 2000; a precipitação; a umidade relativa do ar e a temperatura média mensal de Campo Grande.

L. longipalpis esteve presente ao longo do ano, porém, ressalta-se maior densidade no período subseqüente aos maiores índices pluviométricos registrados no decorrer do ano (Figura 3).

\section{Discussão}

Embora com o uso apenas de CDC não seja possível identificar a fauna flebotomínea em seus aspectos qualitativo e quantitativo, as capturas semanais possibilitaram evidenciar uma grande diversidade com informações quantitativas sobre várias espécies, pois até então, não se conhecia qualquer relato sobre as espécies de flebotomíneos existentes no Município de Campo Grande.

O ano de 1999 foi um ano atípico, com período de seca prolongado o que comprovadamente afeta a densidade dos flebotomíneos. Durante a realização do trabalho ficou demonstrado que há uma grande diversidade de espé- 
Espécies de flebotomíneos coletados com CDC, sexo e ecótopos. Campo Grande, Mato Grosso do Sul, Brasil, fevereiro de 1999 a fevereiro de 2000.

\begin{tabular}{|c|c|c|c|c|c|c|c|c|c|c|c|c|c|c|c|c|c|c|}
\hline \multirow[t]{3}{*}{ Espécies } & \multicolumn{6}{|c|}{ Mata do Zé Pereira } & \multicolumn{6}{|c|}{$\begin{array}{l}\text { Centro de Reabilitação } \\
\text { de Animais Silvestres }\end{array}$} & \multicolumn{6}{|c|}{ Jardim Botânico } \\
\hline & \multicolumn{2}{|c|}{ Copa } & \multicolumn{2}{|c|}{ Solo } & \multicolumn{2}{|c|}{ Margem } & \multicolumn{2}{|c|}{ Copa } & \multicolumn{2}{|c|}{ Solo } & \multicolumn{2}{|c|}{ Margem } & \multicolumn{2}{|c|}{ Copa } & \multicolumn{2}{|c|}{ Solo } & \multicolumn{2}{|c|}{ Margem } \\
\hline & M & $\mathrm{F}$ & $M$ & $\mathrm{~F}$ & $M$ & $\mathrm{~F}$ & $M$ & $\mathrm{~F}$ & $M$ & $\mathrm{~F}$ & M & $\mathrm{F}$ & $\mathrm{M}$ & $\mathrm{F}$ & M & $\mathrm{F}$ & $M$ & $\mathrm{~F}$ \\
\hline B. avellari & - & - & 3 & - & 1 & - & 3 & - & 32 & - & 8 & - & 1 & - & 2 & - & 4 & - \\
\hline B. brumpti & - & - & - & - & - & - & 1 & - & 1 & - & - & - & 1 & - & 19 & - & 21 & - \\
\hline B. galindoi & - & - & - & - & - & - & 2 & - & 39 & - & 12 & - & - & - & - & - & 1 & - \\
\hline B. pintoi & - & - & 2 & - & - & - & - & - & 3 & - & - & - & - & - & 1 & - & 2 & - \\
\hline Brumptomyia sp. & - & 1 & - & 4 & - & 8 & - & 7 & - & 79 & - & 23 & - & 1 & - & 9 & - & 16 \\
\hline L. aragaoi & - & 2 & 10 & 8 & 17 & 14 & - & - & 4 & 4 & - & 1 & 2 & 4 & 14 & 14 & 17 & 10 \\
\hline L. bourrouli & - & 1 & 2 & 2 & - & - & - & - & - & - & - & - & - & - & - & - & - & - \\
\hline L. campograndensis & - & - & 5 & 2 & 9 & 5 & 1 & - & 2 & - & - & 2 & 1 & - & - & - & - & - \\
\hline L. cerradincola & - & - & - & - & - & - & - & - & - & - & - & - & - & - & - & - & - & - \\
\hline L. christenseni & - & - & - & - & - & 5 & - & 1 & - & 1 & - & 2 & - & - & - & 2 & - & - \\
\hline L. claustrei & - & - & 4 & 8 & - & 4 & - & - & - & - & - & - & - & - & - & - & - & - \\
\hline L. cortelezzii & - & - & - & - & - & 4 & - & - & 8 & 12 & 4 & 3 & - & - & - & - & - & - \\
\hline L. corumbaensis & - & - & - & - & 1 & 1 & - & - & - & 1 & - & - & - & - & - & - & - & - \\
\hline L. cruzi & - & - & - & - & - & - & - & - & - & - & - & - & - & - & - & - & - & - \\
\hline L. damascenoi? & - & - & - & 5 & - & 8 & - & - & - & 8 & - & - & - & - & - & 5 & - & 2 \\
\hline L. flaviscutellata & - & - & 1 & 3 & - & 1 & - & - & - & - & - & - & - & - & - & - & - & - \\
\hline L. hermanlenti & 2 & 2 & 13 & 16 & 5 & 6 & - & - & - & - & - & - & - & - & - & - & - & - \\
\hline L. lenti & - & - & 1 & 2 & 1 & 1 & - & - & - & 1 & - & - & 1 & - & 2 & 2 & 2 & 1 \\
\hline L. longipalpis & - & - & - & - & - & - & - & - & - & - & - & - & - & - & - & - & - & - \\
\hline L. longipennis & 1 & 3 & 3 & 2 & 44 & 50 & - & - & 1 & - & - & - & - & - & - & - & - & - \\
\hline L. migonei & - & - & - & - & - & - & - & - & - & - & - & - & - & - & - & - & - & - \\
\hline L. punctigeniculata & - & - & - & - & - & 1 & - & - & - & - & - & - & - & - & - & - & - & - \\
\hline L. quinquefer & 1 & - & 2 & - & 3 & 3 & - & - & - & 1 & - & - & - & - & - & - & - & - \\
\hline L. renei & - & - & - & - & - & - & - & - & - & - & 1 & - & - & - & - & - & - & - \\
\hline L. shannoni & 2 & 1 & 2 & - & 2 & 1 & - & - & - & 1 & - & - & - & - & - & 3 & - & 1 \\
\hline L. sordellii & - & - & 1 & 1 & - & 2 & - & - & - & - & - & - & - & - & 1 & - & - & 1 \\
\hline L. teratodes & - & - & - & - & - & 2 & - & - & - & - & - & - & - & - & - & 5 & - & - \\
\hline L. termitophila & - & - & - & - & - & - & - & - & - & - & - & - & - & - & - & 2 & - & - \\
\hline L. whitmani & - & - & 1 & - & - & 2 & - & - & - & 1 & - & - & - & 1 & 1 & - & - & - \\
\hline Total & 6 & 9 & 50 & 49 & 81 & 102 & 7 & 1 & 90 & 30 & 25 & 8 & 6 & 5 & 40 & 33 & 49 & 15 \\
\hline
\end{tabular}

(continua)

cies no Município de Campo Grande. Dentre os 1.245 flebotomíneos capturados (Tabela 1), $L$. termitophila predominou, seguida por L. aragaoi, L. lenti, L. longipennis e L. longipalpis. L. termitophila e L. lenti que foram mais abundantes no galinheiro, demonstrando estarem adaptadas a ambientes modificados como já assinalado em outras regiões do Mato Grosso do Sul (Galati et al., 1996) e em outros Estados (Gomes \& Galati, 1978). Em virtude da presença constante de L. aragaoi nas três matas estudadas, confirma-se a preferência dessa espécie por ambientes silvestres. Constatamos que 37,6\% dos flebotomíneos capturados nas matas são pertencentes ao gênero Brumptomyia, confirmando seu comportamento selvático como já observado por outros autores (Fraiha et al., 1970), e, ainda, a diversidade de espécies deste gênero.

Houve um aumento considerável no número de exemplares capturados após meses de altos índices pluviométricos, reforçando a hipótese de que a elevação da umidade do meio ambiente em decorrência das chuvas tem como conseqüência a emergência de formas aladas (Forattini, 1973). Destaca-se que, em razão de problemas técnicos no decorrer das capturas, só foi possível de avançar apenas uma semana no mês de fevereiro de 2000, justificando o baixo número de espécimens coletados nes- 
Espécies de flebotomíneos coletados com CDC, sexo e ecótopos. Campo Grande, Mato Grosso do Sul, Brasil, fevereiro de 1999 a fevereiro de 2000.

\begin{tabular}{|c|c|c|c|c|c|c|c|c|c|c|c|c|c|c|}
\hline \multirow[t]{3}{*}{ Espécies } & \multicolumn{6}{|c|}{ Chácara das Palmeiras } & \multicolumn{6}{|c|}{ Centro } & \multicolumn{2}{|c|}{ Total } \\
\hline & \multicolumn{2}{|c|}{ Galinheiro 1} & \multicolumn{2}{|c|}{ Galinheiro 2} & \multicolumn{2}{|c|}{ Galinheiro 3} & \multicolumn{2}{|c|}{ Peridomicílio 1} & \multicolumn{2}{|c|}{ Peridomicílio 2} & \multicolumn{2}{|c|}{ Peridomicílio 3} & \multirow[b]{2}{*}{$\mathrm{M}$} & \multirow[b]{2}{*}{$\mathrm{F}$} \\
\hline & M & $\mathrm{F}$ & M & $\mathrm{F}$ & M & $\mathrm{F}$ & M & $\mathrm{F}$ & M & $\mathrm{F}$ & M & $\mathrm{F}$ & & \\
\hline B. avellari & - & - & 1 & - & 1 & - & - & - & - & - & - & - & 56 & - \\
\hline B. brumpti & - & - & 1 & - & - & - & - & - & - & - & - & - & 44 & - \\
\hline B. galindoi & - & - & - & - & - & - & - & - & - & - & - & - & 54 & - \\
\hline B. pintoi & - & - & - & - & - & - & - & - & - & - & - & - & 8 & - \\
\hline Brumptomyia sp. & - & - & - & - & - & - & - & - & - & - & - & 2 & - & 150 \\
\hline L. aragaoi & - & 2 & - & - & 1 & - & - & - & - & - & - & - & 66 & 59 \\
\hline L. bourrouli & - & - & - & - & - & - & - & - & - & - & - & - & 2 & 3 \\
\hline L. campograndensis & - & - & - & 2 & - & - & - & - & - & - & - & - & 18 & 11 \\
\hline L. cerradincola & - & - & - & 1 & - & - & - & - & - & - & - & - & - & 1 \\
\hline L. christenseni & - & - & - & 3 & - & - & - & - & - & - & - & - & - & 16 \\
\hline L. claustrei & - & - & - & - & - & 1 & - & - & - & - & - & - & 4 & 13 \\
\hline L. cortelezzii & - & 1 & - & 2 & - & - & - & 3 & - & - & - & - & 17 & 25 \\
\hline L. corumbaensis & - & - & - & 1 & - & - & - & - & - & - & - & - & 1 & 3 \\
\hline L. cruzi & - & - & 1 & - & - & - & - & - & - & - & - & - & 1 & - \\
\hline L. damascenoi? & - & 1 & - & - & - & 1 & - & - & - & - & - & - & - & 30 \\
\hline L. flaviscutellata & - & - & - & 1 & - & - & - & - & - & - & - & - & 1 & 5 \\
\hline L. hermanlenti & - & - & - & - & 1 & - & - & - & - & - & - & - & 21 & 24 \\
\hline L. lenti & 5 & 8 & 36 & 37 & 10 & 6 & 1 & - & - & - & - & - & 59 & 58 \\
\hline L. longipalpis & - & - & 13 & 1 & 3 & - & 7 & 2 & 2 & 3 & 27 & 13 & 52 & 19 \\
\hline L. longipennis & - & - & - & - & - & 1 & - & - & - & - & - & - & 49 & 56 \\
\hline L. migonei & - & - & - & 1 & - & - & - & - & - & - & - & - & - & 1 \\
\hline L. punctigeniculata & 1 & 1 & - & 1 & - & - & - & - & - & - & - & - & 1 & 3 \\
\hline L. quinquefer & - & - & - & - & - & - & - & - & - & - & - & - & 6 & 4 \\
\hline L. renei & - & - & - & - & - & - & - & - & - & - & - & - & 1 & - \\
\hline L. shannoni & 17 & 5 & 23 & 3 & 3 & 1 & - & - & - & - & - & - & 49 & 16 \\
\hline L. sordellii & - & - & 1 & 1 & 1 & - & 15 & 17 & - & 1 & 1 & 1 & 20 & 24 \\
\hline L. teratodes & - & - & - & 5 & - & - & - & - & - & - & - & - & - & 12 \\
\hline L. termitophila & 1 & 2 & 61 & 70 & 15 & 23 & - & - & - & 1 & - & - & 77 & 98 \\
\hline L. whitmani & 1 & - & - & - & - & - & - & - & - & - & - & - & 3 & 4 \\
\hline Total & 25 & 20 & 137 & 127 & 35 & 33 & 23 & 22 & 2 & 5 & 28 & 16 & 610 & 635 \\
\hline
\end{tabular}

$M=$ masculino; $F=$ feminino

te período, o que foi corrigido com a média de Williams.

As coletas com armadilha CDC, semanalmente, nos vários ecótopos, foram ricas em espécies, excetuando-se as capturas, na região Central da cidade, que apresentaram somente quatro espécies do gênero Lutzomyia: L. longipalpis, L. sordellii, L. termitophila e L. cortelezzii. Das quatro espécies citadas, L. longipalpis foi a que predominou nesta área, confirmando sua preferência por ambientes antrópicos. Como é comprovadamente vetora da leishmaniose visceral em várias regiões do Brasil (Grimaldi Jr. et al., 1989) e com capacidade adaptativa aos ecótopos artificiais, a sua presença sempre foi uma preocupação (Oliveira et al., 2000), uma vez que já existiam relatos de casos autóctones caninos da doença no Município (Silva et al., 2000).

A flutuação populacional de L. longipalpis foi comprometida pelo pequeno número de exemplares capturados ao longo do ano, todavia, vale frisar a ocorrência de um pico expressivo de captura em janeiro de 2000, possivelmente em decorrência do alto índice pluviométrico nos meses antecedentes.

Destaca-se o encontro de L. longipalpis e $L$. cruzi em simpatria, sendo este o segundo relato da ocorrência destas duas espécies em um mesmo ecótopo no Estado de Mato Grosso do 
Distribuição dos flebotomíneos coletados com CDC, mensalmente, de fevereiro de 1999 a fevereiro de 2000 . Campo Grande, Estado de Mato Grosso do Sul, Brasil.

\begin{tabular}{|c|c|c|c|c|c|c|c|c|c|c|c|c|c|c|}
\hline \multirow[t]{2}{*}{ Espécies } & \multicolumn{11}{|c|}{1999} & \multicolumn{2}{|c|}{2000} & \multirow[t]{2}{*}{ Total } \\
\hline & FEV & MAR & $A B R$ & MAl & JUN & JUL & AGO & SET & OUT & NOV & DEZ & JAN & FEV & \\
\hline B. avellari & 1 & 5 & 2 & 2 & 1 & 1 & 9 & 2 & 5 & 6 & 13 & 9 & - & 56 \\
\hline B. brumpti & 2 & 4 & 1 & - & 1 & 1 & 1 & 3 & - & - & 6 & 25 & - & 44 \\
\hline B. galindoi & - & 5 & 4 & - & 1 & - & - & - & 2 & 2 & 26 & 4 & - & 54 \\
\hline B. pintoi & - & - & - & - & - & - & 2 & - & 1 & - & 3 & 2 & - & 8 \\
\hline Brumptomya sp. & 5 & 11 & 7 & 8 & 2 & 2 & 1 & 1 & 17 & 12 & 52 & 32 & - & 150 \\
\hline L. aragaoi & 17 & 10 & 20 & 11 & 4 & 3 & 2 & 2 & 5 & 4 & 7 & 39 & 1 & 125 \\
\hline L. bourrouli & - & - & - & - & - & - & - & - & 1 & - & 1 & 2 & 1 & 5 \\
\hline L. campograndensis & 4 & 4 & 1 & - & 2 & - & 4 & 4 & - & 2 & 4 & 4 & - & 29 \\
\hline L. cerradincola & - & - & - & - & - & - & - & - & - & - & - & 1 & - & 1 \\
\hline L. christenseni & 1 & 1 & 1 & 2 & 5 & - & - & - & 3 & 1 & 1 & 1 & - & 16 \\
\hline L. claustrei & - & 1 & 9 & 1 & - & 4 & 1 & - & 1 & - & - & - & - & 17 \\
\hline L. cortelezzi & - & - & - & - & - & - & - & - & 4 & 1 & 14 & 22 & 1 & 42 \\
\hline L. corumbaensis & 1 & 2 & - & - & - & - & - & - & - & - & - & 1 & - & 4 \\
\hline L. cruzi & - & - & - & 1 & - & - & - & - & - & - & - & - & - & 1 \\
\hline L. damascenoi? & 1 & - & 2 & 1 & 2 & - & 1 & - & 7 & 3 & - & 12 & 1 & 30 \\
\hline L. flaviscutellata & - & - & - & - & 1 & 2 & 2 & - & - & - & - & 1 & - & 6 \\
\hline L. hermanlenti & 6 & 5 & 2 & 1 & 13 & 1 & 7 & 2 & - & 5 & 1 & 2 & - & 45 \\
\hline L. lenti & 34 & 14 & 10 & 3 & - & 2 & 4 & 6 & 3 & 3 & 13 & 18 & 7 & 117 \\
\hline L. longipalpis & 5 & 2 & 8 & 5 & 2 & - & 8 & 1 & - & 1 & 8 & 31 & - & 71 \\
\hline L. longipennis & 6 & 1 & 6 & 4 & - & - & 2 & 2 & 8 & 5 & 46 & 22 & 3 & 105 \\
\hline L. migonei & - & - & - & - & - & - & - & - & - & - & - & - & 1 & 1 \\
\hline L. punctigeniculata & - & - & 1 & - & - & - & - & - & - & 1 & 1 & - & 1 & 4 \\
\hline L. quinquefer & 1 & - & 2 & 1 & - & - & - & 1 & 4 & 1 & - & - & - & 10 \\
\hline L. renei & - & - & - & - & - & - & - & - & 1 & - & - & - & - & 1 \\
\hline L. shannoni & 5 & 1 & 3 & 4 & 4 & - & 4 & 1 & 4 & 3 & 4 & 28 & 4 & 65 \\
\hline L. sordellii & 1 & - & 1 & 1 & 1 & - & - & - & 2 & - & 2 & 35 & 1 & 44 \\
\hline L. teratodes & - & - & - & 1 & - & - & - & - & 1 & - & 5 & 5 & - & 12 \\
\hline L. termitophila & 33 & 16 & 11 & 16 & 11 & 4 & 1 & 7 & 6 & 3 & 21 & 45 & 4 & 175 \\
\hline L. whitmani & 1 & - & - & 1 & - & - & 1 & 1 & 1 & 1 & - & - & 1 & 7 \\
\hline Total & 124 & 82 & 91 & 63 & 50 & 20 & 50 & 33 & 76 & 64 & 228 & 338 & 26 & 1.245 \\
\hline
\end{tabular}

Sul (E. A. B. Galati, comunicação pessoal). Tal fato reveste-se de importância epidemiológica porque L. cruzi foi recentemente incriminada como vetora da LV no Município de Corumbá (Santos et al., 1998).

L. whitmani foi capturada com mais freqüência na mata e no galinheiro, demonstrando que esta espécie deve estar em um processo de adaptação ao ambiente antrópico como demonstrado em outras áreas do Brasil (Azevedo et al., 1996; Azevedo \& Rangel, 1991; Brazil et al., 1991; Forattini, 1960). Apesar da captura de poucos exemplares, é importante observar que este flebotomíneo é tido como o principal vetor de Leishmania (Viannia) braziliensis em diversas regiões do país (Mayrink et al., 1979; Queiroz et al., 1991), estando, também, associado à transmissão de L. (V.) guyanensis e L. (V.) shawi (Lainson et al., 1989).

A presença de L. shannoni foi significativa, pois esteve presente praticamente em todos os ecótopos, excetuando-se o centro da cidade. Foi capturada em todos os meses do ano, aumentando sensivelmente no mês de janeiro, provavelmente por causa dos altos índices pluviométricos dos meses anteriores. Esta espécie tem sido capturada picando o homem em diversas regiões, sendo considerada altamente antropofílica no Estado da Bahia (Vexenat et al., 1986); no entanto, em área de cerradão no Estado de Mato Grosso do Sul, representou apenas $2,1 \%$ em capturas com isca humana (Galati et al., 1996). Ainda que não seja considerada vetora de leishmaniose, tem sido incriminada 
Precipitação mensal $(\mathrm{mm})$, temperatura média mensal $\left({ }^{\circ} \mathrm{C}\right)$ e distribuição mensal (média de Williams, $\overline{\mathrm{X}} \mathrm{w}$ ) do total de espécimens de flebotomíneos capturados em todos os ecótopos no período de janeiro/1999 a fevereiro/2000. Campo Grande, Mato Grosso do Sul, Brasil.

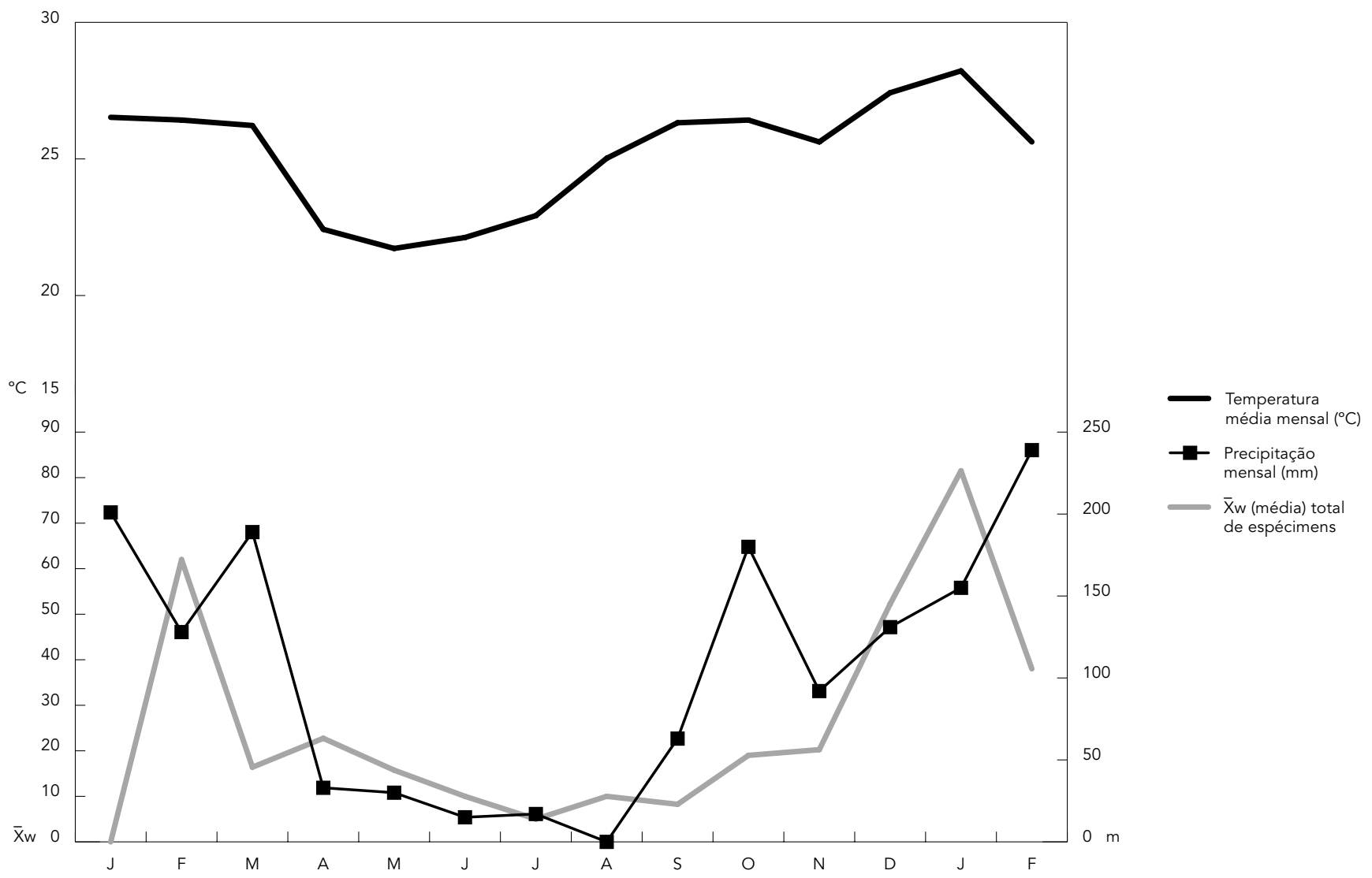

principalmente por conta da transmissão de arboviroses (Comer et al., 1990).

Foram encontrados alguns espécimens de L. flaviscutellata na Mata do Zé Pereira, entretanto, um exemplar foi capturado no galinheiro que se encontrava próximo a uma mata na Chácara das Palmeiras. Esta espécie, que é considerada altamente zoofílica, predomina especialmente em área florestal onde transmite, no nível de solo, Leishmania (L.) amazonensis entre roedores. É julgada uma espécie resistente a mudanças ecológicas, sobrevivendo em áreas de reflorestamento (Ready et al., 1983).

L. campograndensis (Oliveira et al., 2001) descrita a partir deste trabalho, já apresentava uma ampla distribuição geográfica sendo, porém, identificada como L. lutziana. As duas es- pécies são muito próximas, diferindo apenas no comprimento e na extremidade do filamento genital nos machos e, nas fêmeas, no aspecto das espermatecas. Não há informações sobre o comportamento dessa espécie; contudo, ela apresenta associação com área de mata na região de Campo Grande onde esteve presente praticamente em todos os meses do ano.

A respeito de $L$. damascenoi, que foi citada como interrogada, deve-se ao fato de terem sido encontrados somente exemplares fêmeas, ficando muito difícil separá-la de L. christense$n i$ que também esteve representada por exemplares fêmeas, neste caso, separadas pelo tamanho dos dentes posteriores (horizontais). De acordo com Young \& Duncan (1994), L. damascenoi não está presente no Estado de Mato Grosso do Sul; logo, sua existência neste traba- 
Figura 3

Precipitação mensal $(\mathrm{mm})$, temperatura média mensal $\left({ }^{\circ} \mathrm{C}\right)$ e distribuição mensal (média de Williams, $\overline{\mathrm{X}} \mathrm{w}$ ) de Lutzomyia longipalpis capturados em todos os ecótopos no período de fevereiro/1999 a fevereiro/2000. Campo Grande, Mato Grosso do Sul, Brasil.

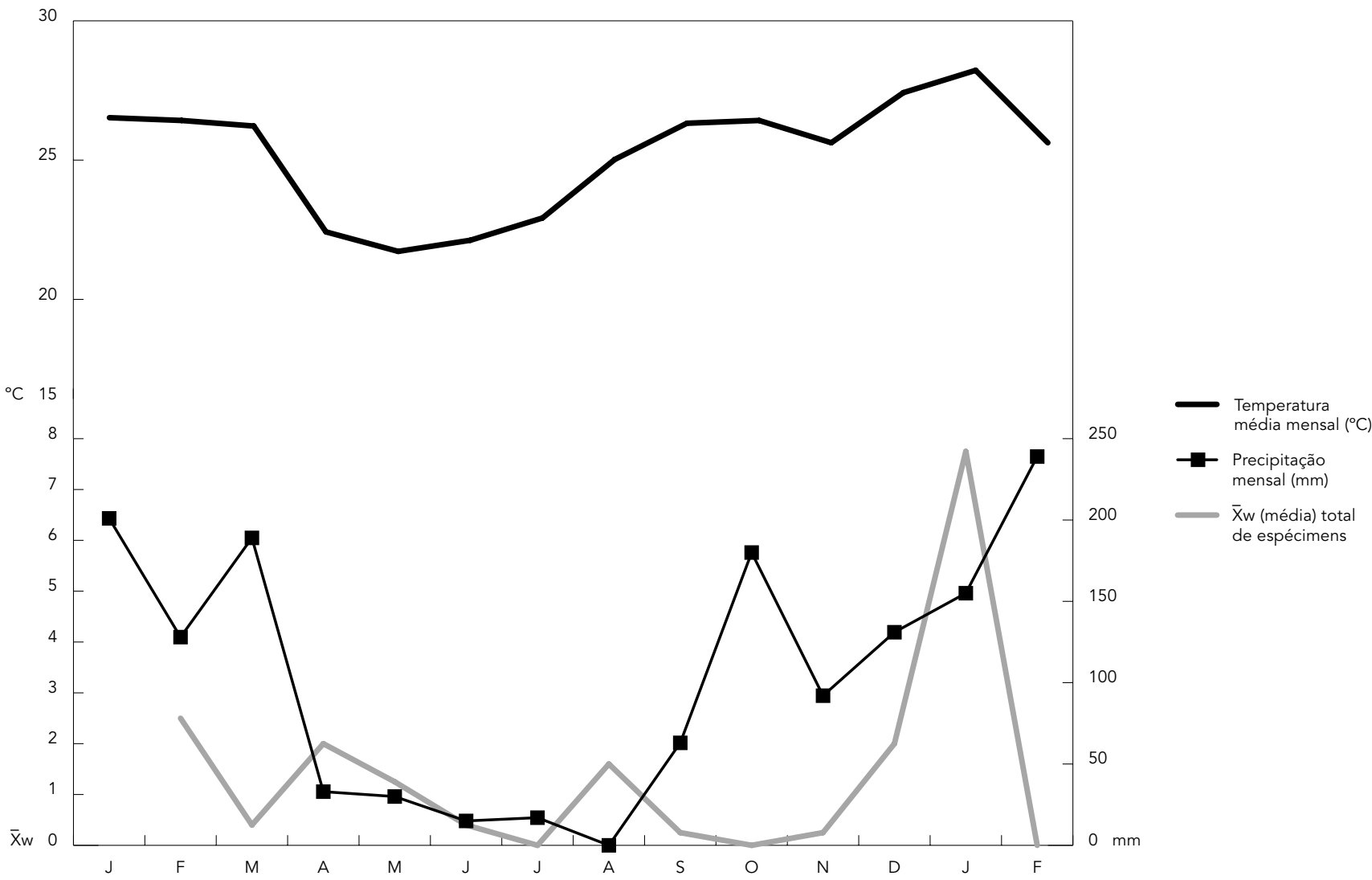

lho requer confirmação mediante novas capturas em que haja a presença de machos.

Em que pese a distribuição geográfica apresentada por Young \& Duncan (1994) não incluir L. cortelezzii no Estado de Mato Grosso do Sul, foi confirmada a sua presença no Município de Campo Grande por intermédio da identificação morfológica criteriosa e comparativa com material de países vizinhos depositados na coleção do Centro de Pesquisas René Rachou, Fundação Oswaldo Cruz.

A razão machos/fêmeas (Tabela 3) das espécies coletadas encontra-se muito próxima de 1/1, com exceção de L. longipalpis e L. shanno$n i$ que foi superior a $2 / 1$. Se o método da coleta estiver refletindo a dinâmica das populações, pode-se pensar que esteja havendo atração diferenciada para ambos os sexos destas espécies como observado por Barretto (1943) e Aguiar et al. (1985) num estudo em que assinalam que capturas com armadilhas luminosas têm a vantagem de oferecer um número maior de machos. Ou então, as armadilhas foram colocadas próximas aos criadouros, e é sabido que os machos nascem antes das fêmeas. Há também a possibilidade de que os machos sejam atraídos às armadilhas quando do acompanhamento às fêmeas para a cópula, pois se sabe que, machos de flebotomíneos formam agregados com o propósito de acasalamento (Feliciangeli, 1987).

As espécies L. cortelezzii, L. claustrei, L. renei e L. flaviscutellata são assinaladas pela primeira vez em Mato Grosso do Sul. 


\section{Agradecimentos}

Ao Conselho Nacional de Desenvolvimento Científico e Tecnológico, à Secretaria Municipal de Saúde de Campo Grande, à Fundação Nacional de Saúde (FUNASA) e à Universidade Federal de Mato Grosso do Sul (UFMS) pelo suporte financeiro. Ao Dr. Francisco Gonçalves de Carvalho (Centro de Controle de Zoonoses), Dra. Elaine Araújo e Silva (Laboratório de Entomologia), aos técnicos José Fernandes da Silva (FUNASA), Fernando Honorato do Prado (FUNASA) e Miriam da Silva Santos (in memorian), Dra. Elisabete Friozi (Centro de Controle de Zoonoses), Dra. Eunice Aparecida Bianchi Galati (Faculdade de Saúde Pública, Universidade de São Paulo), Profa. Maria Elizabeth Cavalheiros Dorval (UFMS) e Profa. Elisa Teruya Oshiro (UFMS) pela ajuda técnica durante nosso trabalho.

\section{Referências}

AGUIAR, G. M.; VILELA, M. L.; SCHUBACK, P.; SOUCASAUX, T. \& AZEVEDO, A. C. R., 1985. Aspectos da ecologia dos flebótomos do Parque Nacional da Serra dos Órgãos, Rio de Janeiro. IV. Freqüência mensal em armadilhas luminosas (Diptera, Psychodidae, Phlebotominae). Memórias do Instituto Oswaldo Cruz, 80:465-482.

AZEVEDO, A. C. R. \& RANGEL, E. F., 1991. A study of sandfly species (Diptera, Psychodidae; Phlebotominae) in a focus of cutaneous leishmaniasis in the municipality of Baturité, Ceará, Brazil. Memórias do Instituto Oswaldo Cruz, 86:405-410.

AZEVEDO, A. C. R.; VILELA, M. L.; SOUZA, N. A.; ANDRADE-COELHO, C. A.; BARBOSA, A. F; FIRMINO, A. L. S. \& RANGEL, E. F., 1996. The sand fly fauna (Diptera, Psychodidae: Phlebotominae) of a focus of cutaneous leishmaniasis in Ilhéus, State of Bahia, Brazil. Memórias do Instituto Oswaldo Cruz, 91:75-79.

BARRETTO, M. P., 1943. Observações sobre a Biologia, em Condições Naturais, dos Flebótomos do Estado de São Paulo (Diptera, Psychodidae). São Paulo: Tipografia Rossolillo.

BRAZIL, R. P.; MORTON, I. E. \& WARD, R. D., 1991. Notes of the feeding habits of Lutzomyia (Nyssomyia) whitmani (Diptera: Psychodidae) in Ceará State, Northeast Brazil. Memórias do Instituto Oswaldo Cruz, 86:497-498.

COMER, J. A.; TESH, R. B.; MODI, G. B.; CORN, J. L. \& NETTLES, V. F., 1990. Vesicular stomatitis virus, New Jersey sorotype: Replication in and transmission by Lutzomyia shannoni (Diptera: Psychodidae). American Journal of Tropical Medicine and Hygiene, 42:483-490.

FELICIANGELI, M. D., 1987. Ecology of sandflies (Diptera: Psychodidae) in a restricted focus of cutaneous leishmaniasis in northern Venezuela. I. Description of the study area, catching methods and species composition. Memórias do Instituto Oswaldo Cruz, 82:119-124.
FORATTINI, O. P., 1960. Novas observações sobre a biologia de flebótomos em condições naturais (Diptera: Psychodidae). Arquivo da Faculdade de Higiene e Saúde Pública da Universidade de São Paulo, 25:209-215.

FORATTINI, O. P., 1973. Entomologia Médica. v. 4. São Paulo: Edgard Blücher/Edusp.

FRAIHA, H.; SHAW, J. J. \& LAINSON, R., 1970. Phlebotominae brasileiros 1: Descrição de uma nova espécie de Brumptomyia e chave para identificação dos machos das espécies do gênero (Diptera: Psychodidae). Revista Brasileira de Biologia, 30:465470.

GALATI, E. A. B.; NUNES, V. L. B.; DORVAL, M. E. C.; OSHIRO, E. T.; CRISTALDO, G.; ESPÍNDOLA, M. A.; ROCHA, H. C. \& GARCIA, W. B., 1996. Estudo dos flebotomíneos (Diptera: Psychodidae), em área de leishmaniose tegumentar, no Estado de Mato Grosso do Sul, Brasil. Revista de Saúde Pública, 30:115-128.

GOMES, A. C. \& GALATI, E. A. B., 1978. Flebotomíneos encontrados em galinheiros experimentais no Estado de São Paulo e Minas Gerais (Brasil) e algumas observações ecológicas. Revista de Saúde Pública, 12:403-407.

GRIMALDI Jr., G.; TESH, R. B. \& McMAHON-PRATT, D., 1989. A review of the geographic distribution and epidemiology of leishmaniasis in the New World. American Journal of Tropical Medicine and Hygiene, 41:687-725.

HADDOW, A. J., 1954. Studies on the biting habits of African mosquitoes: An appraisal of methods employed with special reference to the twenty-four hours catch. Bulletin of Entomological Research, 45:199-242.

HADDOW, A. J., 1960. Studies on the biting habits and medical importance of East African mosquitoes in the genus Aedes. I subgenera Aedimorphus, Banksinella and Nunnius. Bulletin of Entomological Research, 50:759-779. 
IBGE (Fundação Instituto Brasileiro de Geografia e Estatística), 1991. Censo Demográfico. Resultados do Universo Relativos às Características da População e dos Domicílios. Número 25, Mato Grosso do Sul. Rio de Janeiro: IBGE.

LAINSON, R.; BRAGA, R. R.; SOUZA, A. A.; POVOA, M. M.; ISHIKAWA, A. Y. \& SILVEIRA, F. T., 1989. Leishmania (Viannia) shawi sp. N., a parasite of monkeys, sloths and procyonids in Amazonian Brazil. Annales de Parasitologie Humane et Comparé, 64:200-207.

MARGALEF, R., 1974. Ecología. Barcelona: Omega.

MAYRINK, W.; WILLIAMS, P.; COELHO, M. V.; DIAS, M. \& MARTINS, A. V., 1979. Epidemiology of dermal leishmaniasis in the Rio Doce Valley, State of Minas Gerais, Brazil. Annals of Tropical Medicine and Parasitology, 73:123-137.

OLIVEIRA, A. G.; ANDRADE, J. D.; FALCÃO, A. L. \& BRAZIL, R. P., 2001. A new sand fly, Lutzomyia campograndensis sp. n. (Diptera: Psychodidae: Phlebotominae) from the State of Mato Grosso do Sul, Brazil. Memórias do Instituto Oswaldo Cruz, 96:325-329.

OLIVEIRA, A. G.; FALCÃO, A. L. \& BRAZIL, R. P., 2000. Primeiro encontro de Lutzomyia longipalpis (Lutz \& Neiva, 1912) na área urbana de Campo Grande, MS, Brasil. Revista de Saúde Pública, 34:654-655.

PREFEITURA MUNICIPAL DE CAMPO GRANDE, 1995. Instituto Municipal de Planejamento Urbano de Campo Grande (PLANURB). 3a Ed. Campo Grande: Prefeitura Municipal de Campo Grande.

QUEIROZ, R. G.; VASCONCELOS, A. W.; VASCONCELOS, I. A. B.; SOUZA, R. N.; PESSOA, F. A. C.; ALENCAR, J. E. \& DAVID, J. R., 1991. Phlebotomine sandfly (Diptera: Psychodidae) fauna survey in an American cutaneous leishmaniasis (ACL) focus in Baturité, Ceará State Northeast Brazil. Parassitologia, 33(Sup. 1):159-167.
READY, P. D.; LAINSON, R. \& SHAW, J. J., 1983. Leishmaniasis in Brazil: XX. Prevalence of "enzootic rodent leishmaniasis" (Leishmania mexicana amazonensis), and apparent absence of "pian bois" (Le. braziliensis guyanensis), in plantations of introduced tree species and in the other non-climax forests of eastern Amazonia. Transactions of the Royal Society of Tropical Medicine and Hygiene, 77:775-785.

SANTOS, S. O.; ARIAS, J.; RIBEIRO, A. A.; HOFFMANN, M. P.; FREITAS, R. A. \& MALACCO, M. A. F., 1998. Incrimination of Lutzomyia cruzi as a vector of American Visceral Leishmaniasis. Medical and Veterinary Entomology, 12:315-317.

SERVICE, M. W., 1993. Mosquito Ecology: Field Sampling Methods. London: Chapman Hall.

SILVA, E. S.; DE CARVALHO, F. G.; SILVA, E. A.; FIOZI, E.; OLIVEIRA, A. G. \& BRAZIL, R. P., 2000. Primeiro relato de leishmaniose visceral canina em área urbana do Município de Campo Grande, Mato Grosso do Sul. Revista da Sociedade Brasileira de Medicina Tropical, 33:318-319.

VEXENAT, J. A.; BARRETTO, A. C.; CUBA, C. A. C. \& MARSDEN, P. D., 1986. Características epidemiológicas da leishmaniose tegumentar americana em uma região endêmica do Estado da Bahia: III. Fauna flebotomínica. Memórias do Instituto Oswaldo Cruz, 81:293-301.

YOUNG, D. G. \& DUNCAN, M. A., 1994. Guide to the Identification and Geographic Distribution of Lutzomyia Sand Flies in Mexico, the West Indies, Central and South America (Diptera: Psychodidae). Gainesville: Associated Publishers.

Recebido em 5 de julho de 2002

Versão final reapresentada em 25 de outubro de 2002 Aprovado em 23 de janeiro de 2003 